\title{
Reduction of Some Fractional Partial Differential Equations with Variable Coefficients and Exact Solutions
}

\section{Nasser S Elazab*}

Faculty of Science, Department of Mathematics, Cairo University, Egypt

\begin{abstract}
It is shown that a fractional evolution equation is reduced to a functional equation with proportional delay. Which in turn can be reduced to partial differential equations with variable coefficients. By using the extended unified method, some exact solutions to the fractional Burger's equation are obtained.
\end{abstract}

Keywords: Space time fractional equation; Partial differential equations; Variable coefficients; Exact solutions

\section{Introduction}

Dynamic evolution (parabolic) equations in biological, chemical, mechanical and physical systems involving fractional derivatives had received the attention of different works in many areas. This may be argued to the fact that the fractional derivatives retain many novel aspects. So that some new phenomena were revealed by the fractional equation modelling. Many studies of fractional equation modelling to these systems were currently studied in the literature, notably for biological and chemical dynamical-system [1-6]. In contrast, up to our knowledge, few works were devoted to the fractional mechanical and physical systems $[7,8]$. This may be due to the fact that in these later systems evolution equations are nonlinear or semilinear and they are of higher order derivatives. The relevant studies are mainly focusing on the numerical treatment to these equations [9-11]. It had been pointed out that anomalous diffusion and anomalous transport, as novel phenomena do occur [1-6].

In the real applications, the Caputo definition for fractional derivative FD is convenient and it is the mainly used in mathematical modelling. Indeed, an observable function is required to have continuous derivatives up to some order " $m$ " with relevance to a given evolution equation. We mention that, the kernel in the definition of the time (or space) FD is a monomial in $\left(\mathrm{t}-\mathrm{t}^{\prime}\right)$ ( or $\left(\mathrm{x}-\mathrm{x}^{\prime}\right)$ ) (say), respectively. In the case of time FD, it retains the effect of the recent history on the growth (or decay) of an observable function " $\mathrm{u}(\mathrm{x}, \mathrm{t})$ ". While in the case of the presence of a general kernel, namely, $K\left(t, t^{\prime}\right)$ it measures the effect of the complete history when the lower bound of the integral extends to $-\infty$ (see 1.1) and when $t$ replaces $\mathrm{x}$. We think that fractional equations modelling are relevant whenever a system has a characteristic time: $\left(\tau_{c}\right)$ which is assumed to be high enough to support a time history. It is worthy to notice that

$$
\tau_{c}=-\lim _{t \rightarrow \infty} \log (|u(x, t)| / t)
$$

When the limit exists $[4,5]$. The notion of the characteristic time may be made clear in what it follows. When considering the fractional equation modelling of an oscillatory system with frequency $\omega$, then, merely we have $\tau_{c}=\omega^{-1}$. So that fractional equation modelling for high frequency oscillators is inadequate. In contrast to the limitation of using time FD in real systems, using the space FD has everywhere a meaningful aspect. For example, when the order of the space derivative is $\alpha$ and $\mathrm{m}-1<\mathrm{a}<\mathrm{m}$, ( cf. (1.1), then the space fractional modelling describes a system that changes its behaviour from being with convection to be diffusive, from being diffusive to be dispersive, or from being dispersive to be ultra-diffusive when $m=1 ; 2 ; 3 ; 4$ respectively. Thus, in this case space-fractional equation modelling shows the behaviour of a system in transient states. So that, it may stand to explain a "strange spacebehaviour" of the dynamical systems.

Now the Caputo fractional derivative is defined for $\mathrm{x}>0$ and for $\mathrm{x}<0$ respectively $[12,13]$;

$$
\begin{aligned}
& D_{x}^{\alpha} f(x)=\frac{1}{\Gamma(m-\alpha)} \int_{0}^{x}\left(x-x_{1}\right)^{-\alpha+m-1} f^{(m)}\left(x_{1}\right) d x_{1} \\
& \left(-D_{x}\right)^{\alpha} f(x)=\frac{(-1)^{m}}{\Gamma(m-\alpha)} \int_{x}^{0}\left(x_{1}-x\right)^{-\alpha+m-1} f^{(m)}\left(x_{1}\right) d x
\end{aligned}
$$

Where, $f \in C_{\alpha}^{m}=\left\{f, f^{(\mathrm{m})} \in C_{\alpha}\right\}$,

And $\mathrm{C}_{\alpha}$ is the space of functions such that the integrals in the right side of (1.1) and (1.2) exist.

\section{Reduction of the Fractional Evolution Equations}

Here, we consider the equation;

$$
\mathrm{u}_{t}(x, t)+a \frac{\partial^{k}}{\partial x^{k}} u(x, t)+b(u(x, t))_{x}^{j}=0
$$

Where $\mathrm{t}>0,-\infty<\mathrm{x}<\infty, \mathrm{k}<\mathrm{n}$

It is worth noticing that (2.1) reduces to Burger's, KdV, modified $\mathrm{KdV}, \mathrm{KdV}$-Burger's, or Kuramoto-Sivashinsky equations when $\mathrm{a}=0$; $n=2 ; j=2, a=0 ; n=3 ; j=2, a=0 ; n=3 ; j=3, a \neq 0 ; k=2 ; n=4 ; j=2$, or $a \neq 0 ; k=2$; $\mathrm{n}=4 ; \mathrm{j}=2$. In this section we shall find two fractional forms to $(2.1)$ and analyse their reduction to partial differential equations (PDES) with variable coefficients.

\section{Reduction to the first form}

We assume $x>0 ; t>0$, and consider the first form to the fractional differential equation for (2.1), namely

$$
\begin{aligned}
& D_{t}^{\alpha} u(x, t)+a D_{x}^{\beta} u(x, t)+D_{x}^{\gamma} u(x, t)+b D_{x}^{\delta} u(x, t)^{j}=0, \\
& 0 \leq \alpha<1, k-1 \leq \beta<k, n-1 \leq \gamma<n, 0 \leq \delta<1
\end{aligned}
$$

*Corresponding author: Nasser S. Elazab, Faculty of Science, Department of Mathematics, Cairo University, Egypt, Tel: 00201148429161; E-mail: elazab@mu.edu.sa

Received February 05, 2018; Accepted February 19, 2018; Published February 26, 2018

Citation: Elazab NS (2018) Reduction of Some Fractional Partial Differentia Equations with Variable Coefficients and Exact Solutions. Int J Swarm Intel Evol Comput 7: 168. doi: 10.4172/2090-4908.1000168

Copyright: ( $) 2018$ Elazab NS, et al. This is an open-access article distributed under the terms of the Creative Commons Attribution License, which permits unrestricted use, distribution, and reproduction in any medium, provided the original author and source are credited. 
Citation: Elazab NS (2018) Reduction of Some Fractional Partial Differential Equations with Variable Coefficients and Exact Solutions. Int J Swarm Intel Evol Comput 7: 168. doi: 10.4172/2090-4908.1000168

We use the FD which is defined in (1.1) into (2.2) and we have the following theorems. Theorem 2.1. The equation (2.2) reduces to the functional equations

$$
\begin{aligned}
& t^{n-\gamma} \frac{\partial}{\partial t y} u(x, t y)+x^{n-\gamma}\left(a \frac{\partial^{k}}{\partial(x y)^{k}} u(y x, t)+\frac{\partial^{n}}{\partial(x y)^{n}} u(x y, t)+b \frac{\partial}{\partial x y}(u(x y, t))^{j}\right)=0 \\
& \text { When } 0<y \leq 1, \alpha=\delta=\gamma-n+1, \beta=\gamma-n+k \text { and } 0<y<1 \\
& t^{1-\alpha} \frac{\partial}{\partial t y} u(x, t y)+a x^{k-\beta}(1-y)^{\alpha-\beta+k-1} \frac{\partial^{k}}{\partial(x y)^{k}} u(y x, t)+x^{n-\gamma}(1-y)^{\alpha-\gamma+n-1} \\
& \left.\frac{\partial^{n}}{\partial(x y)^{n}} u(x y, t)+\mathrm{b} x^{1-\delta}(1-y)^{\alpha-\delta} \frac{\partial}{\partial x y}(u(x y, t))^{j}\right)=0
\end{aligned}
$$

When $\alpha>\operatorname{Max}(\delta, \beta-k+1, \gamma-n+1)$

respectively.

Proof. In (2.2), by setting $\alpha=\delta=\gamma-n+1, \beta=\gamma-n+k$ and by using the transformations $x_{1}=x y, t_{1}=t y$, it becomes;

$$
\begin{aligned}
& \frac{1}{\Gamma(n-\gamma)} \int_{0}^{1}(1-y)^{-\gamma+(\mathrm{n}-1)}\left(t^{n-\gamma} \frac{\partial}{\partial t y} u(x, t y)+a x^{n-\gamma} \frac{\partial^{k}}{\partial(\mathrm{xy})^{k}} u(y x, t)+x^{n-\gamma}\right. \\
& \left.\frac{\partial^{n}}{\partial(x y)^{n}} u(y x, t)+\mathrm{b} x^{n-\gamma} \frac{\partial}{\partial x y}(u(y x, t))^{j}\right) d y=0
\end{aligned}
$$

As the function in the outside of the brackets is strictly increasing in $0 \leq \mathrm{y} \leq 1$, then (2.3) holds when the terms between brackets vanish and we get the functional equation (2.3).

In the case when

$$
\alpha>\operatorname{Max}(\delta, \beta-k+1, \gamma-n+1),
$$

and in a way similar to that done in the above, the equation $(2.2)$ is reduced to (2.4). We mention that functional equations similar to (2.4) can be obtained as a reduction to (2.2) when,

$$
\begin{aligned}
& \alpha>\operatorname{Max}(\delta, \beta-k+1, \gamma-n+1), \beta-k+1>\operatorname{Max}(\delta, \alpha, \gamma-n+1) \\
& \text { or when } \gamma-n+1>\operatorname{Max}(\delta, \alpha, \beta-k+1) .
\end{aligned}
$$

Further reductions of (2.2) to a functional equation for less restrictive conditions on $\alpha, \beta, \gamma, \delta, \mathrm{n}, \mathrm{k}$, can also be done.

It is worth noticing that functional equations (2.3) and (2.4) are PDE's with proportional delay. We may justify this by the following. When considering (1.1) by replacing $\mathrm{x}$ by $\mathrm{t}$ (or namely when replacing the space FD by the time FD) then this shows that the system under consideration retains distributed delays. Indeed, an equation with "distributed" delay can be transformed to another one's with proportional delay by the transformation $t-t^{\prime}=\log \left(T / T_{0}\right)$.

By using the assumption that that $\mathrm{u}(\mathrm{x}, \mathrm{t})$ has a continuous partial derivatives up to $\mathrm{n}$-nth order, then, in (2.3) by taking the limit when $\mathrm{y} \rightarrow 1^{\text {; }}$, we get a PDE's with variable coefficients.

$$
t^{n-\gamma} \frac{\partial}{\partial t} u(x, t)+x^{n-\gamma}\left(a \frac{\partial^{k}}{\partial(x)^{k}} u(x, t)+\frac{\partial}{\partial x}(u(x, t))^{j}\right)=0
$$

\section{Reduction to the second form}

For the second fractional form to (2.1), we proceed by rewriting (1.1) when $\mathrm{m}=1$,

$$
D_{x}^{\alpha} f(x)=\frac{1}{\Gamma(1-\alpha)} \int_{0}^{x}\left(x-x_{1}\right)^{-\alpha} f^{\prime}\left(x_{1}\right) d x_{1}, x>0,0 \leq \alpha<1
$$

In (1.13) by writing $\alpha=\mu-s+1, s-1 \leq \mu<s$ and by using the equality

$$
D_{x}^{\mu} f(x)=D_{x}^{\mu-s+1}\left(D_{x}^{s-1} f(x)\right)=D_{x}^{s-1}\left(D_{x}^{\mu-s+1} f(x)\right)
$$

The first equality gives rise to the definition in (1.1), while the second ones gives rise to,

$$
D_{x}^{\mu} f(x)=\frac{D_{x}^{s-1}}{\Gamma(s-\mu)} \int_{0}^{x}\left(x-x_{1}\right)^{-\mu+s-1} f^{\prime}\left(x_{1}\right) d x_{1}, x>0, s-1 \leq \mu<s
$$

Now by using (2.9) into (2.2), and by the same way as in the above, and when

$$
\alpha=\delta=\gamma-n+1, \beta=\gamma-n+k,
$$

we get for the second reduced form namely the functional equation $t^{n-\gamma} \frac{\partial}{\partial t y} u(x, t y)+a \frac{\partial^{k-1}}{\partial x^{k-1}}\left(x^{n-\gamma} \frac{\partial}{\partial x y} u(y x, t)\right)+\frac{\partial^{n-1}}{\partial x^{n-1}}\left(x^{n-\gamma} \frac{\partial}{\partial x y} u(x y, t)\right)+b x^{n-\gamma} \frac{\partial}{\partial x y}(u(x y, t))^{j}=0$

When $0<y \leq 1, \alpha=\delta=\gamma-n+1, \beta=\gamma-n+k$

We mention the case when

$$
\alpha>\operatorname{Max}(\delta, \beta-k+1, \gamma-n+1), 0<y<1,
$$

we get,

$$
\begin{aligned}
& t^{1-\alpha} \frac{\partial}{\partial x y} u(x, t y)+a(1-y)^{\alpha-\beta+k-1} \frac{\partial^{k-1}}{\partial x^{k-1}}\left(x^{k-\beta} \frac{\partial}{\partial(x y)} u(y x, t)\right)+(1-y)^{\alpha-\gamma+n-1} \\
& \frac{\partial^{n-1}}{\partial x^{n-1}}\left(x^{n-\gamma} \frac{\partial}{\partial(x y)} u(x y, t)\right)+b x^{1-\delta}(1-y)^{\alpha-\delta} \frac{\partial}{\partial x y}(u(x y, t))^{j}=0
\end{aligned}
$$

Equations similar to (2.11) are obtained when,

$\delta>\operatorname{Max}(\alpha, \beta-k+1, \gamma-n+1), \beta-k+1>\operatorname{Max}(\delta, \alpha, \gamma-n+1), \gamma-n+1>\operatorname{Max}(\delta, \alpha, \beta-k+1)$

In (2.10) by taking the limit when $\mathrm{y} \rightarrow 1$, we get the PDE;

$$
t^{n-\gamma} \frac{\partial}{\partial t} u(x, t)+a \frac{\partial^{k-1}}{\partial x^{k-1}}\left(x^{n-\gamma} \frac{\partial}{\partial x} u(x, t)\right)+\frac{\partial^{n-1}}{\partial x^{n-1}}\left(x^{n-\gamma} \frac{\partial}{\partial x} u(x, t)\right)+b x^{n-\gamma} \frac{\partial}{\partial x}(u(x, t))^{j}=0
$$

\section{Fractional equation when $\mathbf{x}<\mathbf{0}$; $\mathbf{t}>\mathbf{0}$}

For fractional equations when $x<0 ; t>0$, the equations (2.1) and (2.2) take respectively the form

$$
\begin{aligned}
& u_{t}(x, t)+(-1)^{k} a \frac{\partial^{k}}{\partial(-x)^{k}} u(x, t)+(-1)^{n} \frac{\partial^{n}}{\partial(-x)^{n}} u(x, t)-b\left(u(x, t)^{j}\right)_{(-x)}=0 \\
& D_{t}^{\alpha} u(x, t)+(-1)^{k} a\left(-D_{x}\right)^{\beta} u(x, t)+(-1)^{n}\left(-D_{x}\right)^{\gamma} u(x, t)-b\left(-D_{x}\right)^{\delta} u(x, t)^{j}=0
\end{aligned}
$$

In this case reduction of FDE's to functional one's is not direct as done in sections 2.1 and 2.2 .

\section{Exact Solution of Fractional Burger's Equation}

The First and second form to the fractional Burger's equation read

$$
\begin{aligned}
& t^{2-\gamma} \frac{\partial}{\partial t} u(x, t)+\frac{\partial}{\partial x}\left(x^{2-\gamma} \frac{\partial}{\partial x} u(x, t)\right)+x^{2-\gamma} u(x, t) \frac{\partial}{\partial x}(u(x, t))^{j}=0 \\
& t^{2-\gamma} \frac{\partial}{\partial t} u(x, t)+\frac{\partial}{\partial x}\left(x^{2-\gamma} \frac{\partial}{\partial x} u(x, t)\right)+x^{2-\gamma} u(x, t) \frac{\partial}{\partial x}(u(x, t))^{j}=0
\end{aligned}
$$

Hereafter, we confine ourselves to find the exact solutions of $(3: 1)_{2}$ by using the extended unified method which had been suggested by the author, in collaboration [14-16]. It generalizes the unified method that unify all methods known in the literature for finding exact traveling wave solutions. By this method the solution of $(3: 1)_{2}$ is mapped to polynomial solutions, or to a rational function in an auxiliary function with first order linear or nonlinear auxiliary equations [17].

\section{Polynomial solutions}

We proceed by writing $\tau=\mathrm{t}^{\gamma-1}$ assume that the solution of $(3: 1)_{2}$ takes the form

$$
u(x, \tau)=\sum_{j=1}^{j=n} a_{j}(x, \tau) \varphi^{j}(x, \tau), \varphi(x, \tau)=\sum_{j=1}^{j k=} c_{j}(x, \tau) \varphi^{j}(x, \tau), \varphi_{\tau}(x, \tau)=\sum_{j=1}^{j k k} b_{j}(x, \tau) \varphi^{j}(x, \tau)
$$

together with compatibility equation $\varphi_{x x}(x, \tau)=\varphi_{x x}(x, \tau)$. It is worth noticing that the last equation splits into $2 \mathrm{k}-1$ equations when $\mathrm{k} \geq 2$, and when substituting from the equation (3.2) into (3:1)2, we get $\mathrm{n}+2(\mathrm{k}-1)+1$ "principle"equations. The symbolic computations were 
Citation: Elazab NS (2018) Reduction of Some Fractional Partial Differential Equations with Variable Coefficients and Exact Solutions. Int J Swarm Intel Evol Comput 7: 168. doi: 10.4172/2090-4908.1000168

used to solve these two sets of equations via Mathematica or by any package. The balance and consistency conditions are respectively given by $n=k-1$ and $2 k \leq 7$. Thus, solutions hold when $k=2,3$.

\subsubsection{When $\mathrm{k}=2$}

We list the steps of computations

Step 1. Solving the principle equations. These equations were solved respectively to get $a_{1}(x, t), b_{i}(x, t), j=2,1,0$.

Step 2. Solving the compatibility equations. They were solved to get $\mathrm{a}_{0}(\mathrm{x}, \mathrm{t})$ and $\mathrm{c}_{0}(\mathrm{x}, \mathrm{t})$, where two equations hold simultaneously.

Step 3. Solving the auxiliary equations, namely $(3: 2)_{2}$ is solved and $(3: 2)_{3}$ has to hold identically.

Step 4 . Finding the exact solution by using $(3: 2)_{1}$ : We found that

$u(x, t)=\frac{2 a \gamma x^{\gamma-1}}{a\left(C_{30}+x^{\gamma}\right)-\tau \gamma}, a=\gamma-1, \tau=t^{\gamma-1}$

where $\mathrm{C}_{30}$ is an arbitrary constant [14-16].

\subsubsection{When $\mathrm{k}=3$}

By repeating the same steps of computations, we find that the solution in this case is

$$
\begin{aligned}
& \frac{2 a C_{40} \gamma^{2} x^{\gamma-1}}{-2+\gamma\left(\left(a\left(C_{30}+x^{\gamma}\right)-\tau \gamma\right) C_{40}+2\right)}, a=\gamma-1, \tau=t^{\gamma-1} \\
& \text { where } \mathrm{C}_{30} \text { and } \mathrm{C}_{40} \text { are constants. }
\end{aligned}
$$

\section{Rational function solutions}

In this case, we assume that the solution of $(3: 1)_{2}$ takes the form

$$
\begin{aligned}
& u(x, \tau)=\frac{\sum_{j=1}^{j=n} p_{j}(x, \tau) \varphi^{j}(x, \tau)}{\sum_{j=1}^{j=r} q_{j}(x, \tau) \varphi^{j}(x, \tau)}, \varphi_{x}(x, \tau)=\sum_{j=1}^{j=k} c_{j}(x, \tau) \varphi^{j}(x, \tau) \\
& \varphi_{x}(x, \tau)=\sum_{j=1}^{j=k} b_{j}(x, \tau) \varphi^{j}(x, \tau), \varphi_{x \tau}(x, \tau)=\varphi_{\tau x}(x, \tau)
\end{aligned}
$$

When substituting from the equation (3.5) into $(3: 1)_{2}$, we get $\mathrm{n}+2(\mathrm{k}-1)+1+2 \mathrm{r}$ "principle" equations. The balance condition is $\mathrm{n}-\mathrm{r}=\mathrm{k}-1$. As $\mathrm{k} \geq 1$, and we require that $\mathrm{n} \leq \mathrm{r}$, then we take $\mathrm{k}=1$, thus $\mathrm{n}=\mathrm{r}$. The consistency condition is $\mathrm{n} \leq 3$.

\subsubsection{When $\mathrm{n}=1$}

In this case we have $r=1$ and two compatibility equations. For convenience we use the transformations

$$
p_{1}(x, \tau)=A(x, \tau) q_{1}(x, \tau), p_{0}(x, \tau)=A(x, \tau) q_{0}(x, \tau)+B^{2}(x, \tau)
$$

When substituting from (3.6) into the principle equations we find that the function $\mathrm{A}(\mathrm{x}, \tau)$ satisfies the equation which it is required to solve it, namely (3.1). That is by using the equation (3.6) into the numerator in (3:5) and expanding, we find that it can be an autoBacklund transformation. In this respect we search for a particular solution $\mathrm{A}(\mathrm{x}, \tau)$ for $(3.1)_{2}$, namely

$$
A(x, \tau)=\frac{\left(A_{0}+x^{\gamma-1}\right)}{\left(\tau+\tau_{0}\right)}
$$

By using the same steps of computation, first the principle equations solve to $b_{j}(x, \tau), j=1,0$ and then find $c_{1}(x, \tau)$. The two compatibility equations were solved to get $c_{0 x x}(x, \tau)$ and $c_{0 x}(x, \tau)$. The compatibility equation between these two partial derivatives and the equation results was solved to get $c_{0 \tau}(x, \tau)$ : The compatibility equation between $c_{0 x}(x, \tau)$ and $c_{0 \tau}(x, \tau)$ is constructed and it solves to $c_{0}(x, \tau)$, where new equation obtained is identically equal to zero. The steps 3 and 4 in the case for polynomial solutions are directly used. We get the solution

$$
u(x, \tau)=\frac{a C_{40}+C_{50}\left(A_{0}+x^{\gamma-1}\right)}{-1+C_{50}\left(\tau+\tau_{0}\right)}
$$

where a is given in (3.4). In the case for finding rational solutions, the computation is very lengthy, the cases when $n=2,3$ will not be considered here. We mention that we have verified that the solutions (3.3), (3.4) and (3.8) satisfy the equation (3:1)

\section{Conclusion}

Fractional evolution equations applied to functional partial differential equations with variable coefficients to get exact solutions. The method applied to fractional Burger's equation and some solutions of this equation found. We think that this work will allow to advance the study of applied analysis to solutions of fractional equations. Also, better viewing the behaviour of these solutions will be amenable that leads to better interpreting phenomena revealed by fractional equation modelling in the applied sciences.

\section{References}

1. Sun HG, Chen W, Quan Chen (2009) Variable order fractional differential operators in anomalous diffusion modeling. Phys A Stat Mech Appl 388: 45864592.

2. Yang LI, Sun DH, Zhao M, Cheng SL, Zhang G, et al. (2018) The influence of continuous historical velocity difference information on micro-cooperative driving stability. Phys A Stat Mech Appl 494: 294-301.

3. Zhao C, Zhao Y, Luo L, Li Y (2011) Fractional modelling method research on education evaluation. J Soft 6: 1-7

4. Tarasov VE, Edelman M (2010) Fractional dissipative standard map. Chaos 20: 023127.

5. Cox S (2015) Math reading list 2015, a survey of the literature. University of Central Florida, USA

6. Yilidrim A, Sezar SA (2010) Analytical solution of linear and nonlinear spacetime fractional reaction diffusion equations. Int J Chem Reactor Eng 8: A10.

7. Chechekin AV, Gonchar VY, Gorenflo R, Korabel N, Sokolov IM (2008) Generalized fractional differential equations for accelerating sub-diffusion and truncated levy flights. Phys Rev 78: 21111-21124.

8. Liu Y (2012) Study on multi-order fractional fokker dynamics. J Frac Calc App 2: $1-8$.

9. Yulita R, Batiha B, Taib M (2016) Solutions of fractional zakharov-kuznetsov equations by fractional complex transform. Int Appl Math Res 5: 24-28.

10. Sevimlican A (2010) An approximation to solution of space and time fractiona telegraph equations by he's variational iteration method. Math Prob Engg 2 1-10.

11. Turut V, Güzel N (2013) On solving partial differential equations of fractional order by using the variational iteration method and multivariate padé approximations. Euro J Pure Appl Math 6: 147-171.

12. Elazab NS (2017) Existence of solutions of economic optimal problems with delay. Far East J Math Sci 101: 443-456.

13. Benchohra M, Hamidi N (2010) Fractional order differential inclusions on the half-line. Survey Math Appl 5: 99-112.

14. Gawad HIA, Othman M, Elazab NS (2013) Exact solutions of time dependent kortewg-de-vries equation. Int J Soft Comp Eng 3: 59-63.

15. Gawad HIA, Osman M, Elazab NS (2013) Exact solutions of space dependen korteweg-de-vries equation, J Phys Soc 3: 59-63.

16. Gawad HIA, Osman M, Elazab NS (2013) Exact solutions of space and time dependent burger's equation. ljbas-ljens 13: 65-68.

17. Gawad HIA (2012) Towards a unified method for exact solutions of evolution equations-An application to reaction diffusion equation with finite memory transport. J Stat Phys 147: 506-518. 